\title{
Monitoring and analysis of special crystal surface waviness
}

\author{
JialiangGuan ${ }^{1, a}$,Xian hui Zhao ${ }^{1, b}$, YongRen ${ }^{1, c}$, Ze hai Qi ${ }^{1, d}$, Wen wen Lu $^{1, e}$ and \\ ShenggenZhu ${ }^{2, f}$ \\ ${ }^{1}$ College of Mechanical Electrical,Beijingn UniversityofTechnology,Beijing100124, China; \\ ${ }^{2}$ Beijing Precision Machinery\&Engineering Research Co.,Ltd.,Beijing101312, China. \\ aguanjl@bjut.edu.cn, ${ }^{\text {b } 1099709122 @ q q . c o m, ~}{ }^{\circ} 659619689 @ q q . c o m,{ }^{f}$ tc.zsg@jcsgy.com
}

Keywords: KDP crystal; Acoustic emission technique; frequency domain; time domain;

\begin{abstract}
Using single point diamond knife and quadratic general regression rotary unitized design to do cutting experiments of special crystals (KDP crystal), and using acoustic emission technique to collect signal of KDP crystal with different surface waviness, measure and analyze the test results. By frequency domain and time domain analys is, it can be obtained that $150-200 \mathrm{KHz}$ can be a characteristic band to monitor KDP crystal surface waviness online and mean-square value can be used an important time Domain feature to monitor the process of KDP crystal surface wavinessstatus .
\end{abstract}

\section{Foreword}

KDP crystal is an excellent non-linear optical element. Currently, large-caliber KDP crystal is the only optical crystal materials can be used as inertial confinement fusion system of high power laser driving means the Pockels cell [1]. Surface roughness, surface waviness and transmitted waves are large caliber KDP crystal 3 hardest to achieve technical indicators [2], and the surface waviness is the most complex factors, indicators of the hardest to control. After the use of the KDP crystal SPDT technology for processing, on the machined surface along the cutting path will form an arc tool marks, It will add a small scale periodic disturbance before transmission wave of KDP crystal, this small-scale phase modulation is nonlinear-growth noise source of strong laser, it is easy to form self-focusing damage in a high-power and is necessary to be suppressed. Therefore, line monitoring for surface wavinessstate in the processing of KDP crystal to guide a great significance for the actual processing.

\section{The introduction of acoustic emission technology and signal acquisition.}

\section{The principle of acoustic emission technology}

Detection principle of Acoustic Emission is shown in figure 1. Elastic waveemitted from acoustic emission source will reach the surface of the pick test workpiece eventually through media and lead to mechanical vibration on the workpiece surface.[3]A sensor mounted on the surface of the workpiece will convert the detect transient displacement into an electrical signal,then through the Acoustic

Emission system, the electrical signals is amplified, processed, recorded and displayed, the will be formed specific parameters and waveform data. Finally, the data will be processed and analyzed to obtain Acoustic Emission characteristic of material.

Using AE technology to collect and analyze the Acoustic Emission signal of surface waviness for KDPin different state,then get the mapping between AE signal characteristics and surface waviness, thus judged the state of the surface waviness when processing by the AE signal . 


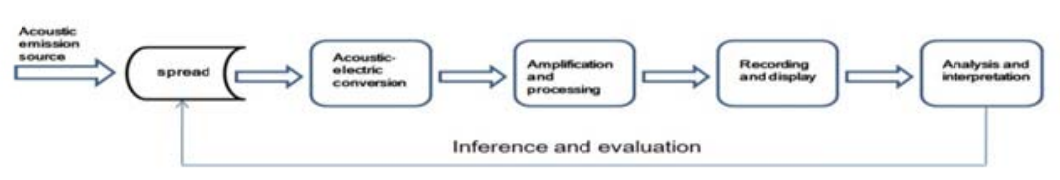

Fig.1 The principle of acoustic emission technology

\section{The collection of experimental data}

\subsection{Set the acoustic emission technology}

The digital filter is set to direct, waveform and parameter thresholds are set to $30 \mathrm{db}$. When formally cutting, the signal of ambient noise and idling spindle willbe filtered preliminary. [4]In trials involving a total of five kinds of machine speed, respectively, 100,275,450,625 and 800r / min.According SPDT principle, the size of the workpiece and the tool rotation radius, a contact time of tool and workpiece respectively is calculated for $131.25 \mathrm{~ms}, 48 \mathrm{~ms}, 19.167 \mathrm{~ms}, 21 \mathrm{~ms}$ and $16.4 \mathrm{~ms}$ at each speed.After the signal of ambient noise and idling spindle are preliminary filtered in the time domain and frequency domain acoustic emission signals, the signal of acoustic emission express a periodic function for tool rotation of the week under various combinations of process parameters, but only when the tool contact with the workpiece, the acoustic emission system will generate signal, so the sampling minimum time should be greater than the contact time of the tool and the workpiece. According to Nyquist sampling theorem, the sampling frequency should be at least twice the highest frequency of the signal,this test sampling frequency is set $3333 \mathrm{KHz}$, the sampling length is selected in accordance with the contact time of the tool and the workpiece at each of the rotational speed. According to the actual situation, choose the parameter interval, locking time, peak interval and storage paths in SAEU2S acoustic emission system software,the acoustic emission signals with different process parameters are collected and stored in the process of KDP crystal.

\subsection{The collection of experimental data}

31 series of tests carried out using the SPDT and quadratic general regression rotary,finally, the surface waviness of each test are shown in Table 1.

\begin{tabular}{llllllllll}
\multicolumn{1}{l}{ Table 1 Quadratic regression rotation experimental design and test result data } \\
\hline Test No & 1 & 2 & 3 & 4 & 5 & 6 & 7 & 8 & 9 \\
\hline Waviness & 7.7 & 17.7 & 14.3 & 19.7 & 7 & 12.2 & 24.3 & 26.3 & 18.6 \\
\hline Test No & 10 & 11 & 12 & 13 & 14 & 15 & 16 & 17 & 18 \\
\hline Waviness & 17.6 & 18 & 14.6 & 14.2 & 15.7 & 20.7 & 19.8 & 10 & 18 \\
\hline Test No & 19 & 20 & 21 & 22 & 23 & 24 & 25 & 26 & 27 \\
\hline Waviness & 7.7 & 30.1 & 21.8 & 52.3 & 12.1 & 9 & 9.1 & 9.3 & 15 \\
\hline Test No & 28 & 29 & 30 & 31 & & & & & \\
\hline Waviness & 11.1 & 14 & 15.9 & 11.9 & & & & & \\
\hline
\end{tabular}

\section{Analysis of acoustic emission signals}

The signal is collected by acoustic emission systems is transient displacement of the workpiece surface, including cutting, machine vibration and environmental noise, large amount of data, monitoring signals can not be directly used to determine the status of KDP crystal surface waviness. [5]Therefore, the acoustic emission signal needs to be processed to obtain a clear signal characteristics which has a corresponding relationship with the surface waviness, realize the online monitoring of KDP crystal surface waviness in the process. The test results are shown in Table 2. 
Table 2 The experiment results grouping of KDP crystal surface waviness

\begin{tabular}{ll}
\hline Wavinessrating & Results Size $(\mathrm{nm})$ \\
\hline Good(Less than 8$)$ & $7 ; 7.7 ; 7.7$ \\
better $(8-14)$ & $9 ; 10 ; 12.1 ; 12.2 ; 12.3$ \\
medium $(14-20)$ & $14.2 ; 14.3 ; 14.6 ; 15.7 ; 17.6 ; 17.7 ; 18 ; 18 ; 18.6 ; 19.7 ; 19.8$ \\
Poor $(20-30)$ & $20.7 ; 21.8 ; 24.3 ; 26.3$ \\
$\begin{array}{l}\text { Very poor (More } \\
\text { than 30) }\end{array}$ & $30.1 ; 52.3$ \\
\hline
\end{tabular}

\subsection{Time domain analysis of acoustic emission signals}

Analyze the acoustic emission signal of time-domain waveform in processing, get the signal changes over time, and determines a characteristic portion of the signal, and overall trends of throughout time domain signal with the increases of waviness.[6] Figure 2 is a single frame of acoustic emission signals in time domain with surface waviness in grades 1-4.0

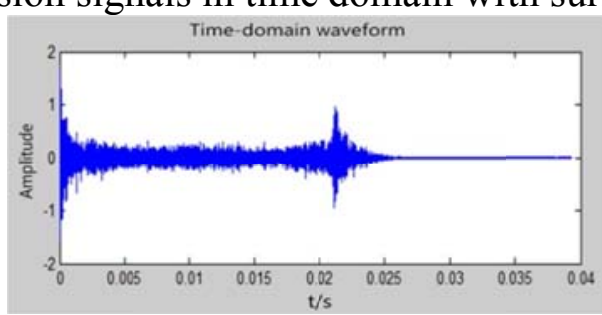

a) Level 1

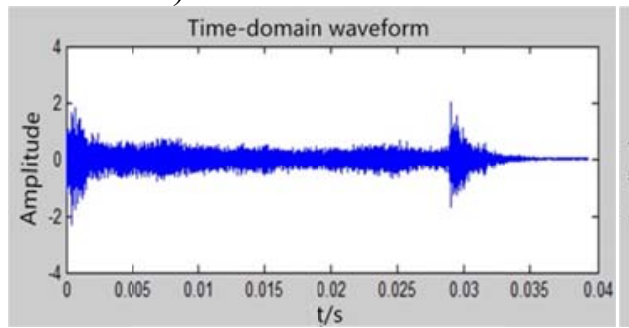

c) Level 1

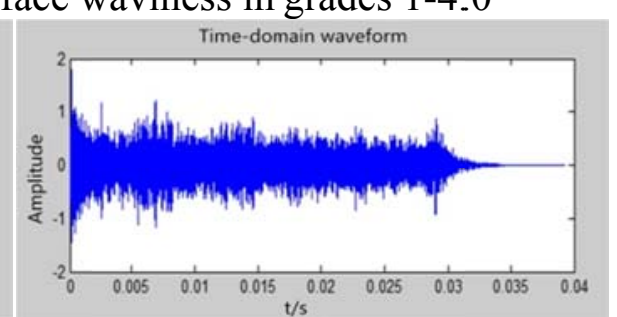

b) Level 2

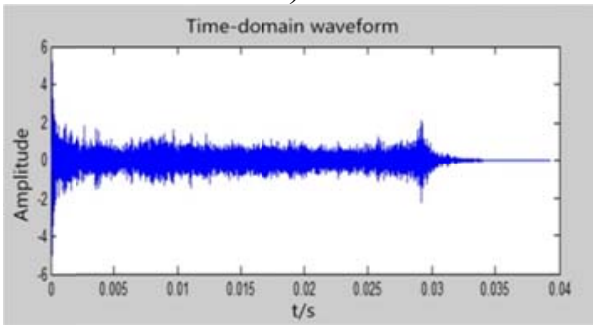

d) Level 1

Fig.2The time-domain waveform of different waviness grade

It can be obtained from Figure 2, the amplitude of signal at the beginning and end are larger in each of the waveform, this is because the energy mutation at tool cuts and cut KDP crystal, It can provide judgments for subsequent frequency domain analysis. Ignore skip signal at both ends, compared to the above-mentioned four graphic show that: With the increase of waviness value, The amplitude of acoustic emission signal in intermediate section has a large overall trend.

According to Time Domain Analysis Method, analyze and calculate Table 2; The time-domain statistical results to draw a line graph, as shown in Figure 3, where the average take its absolute value.

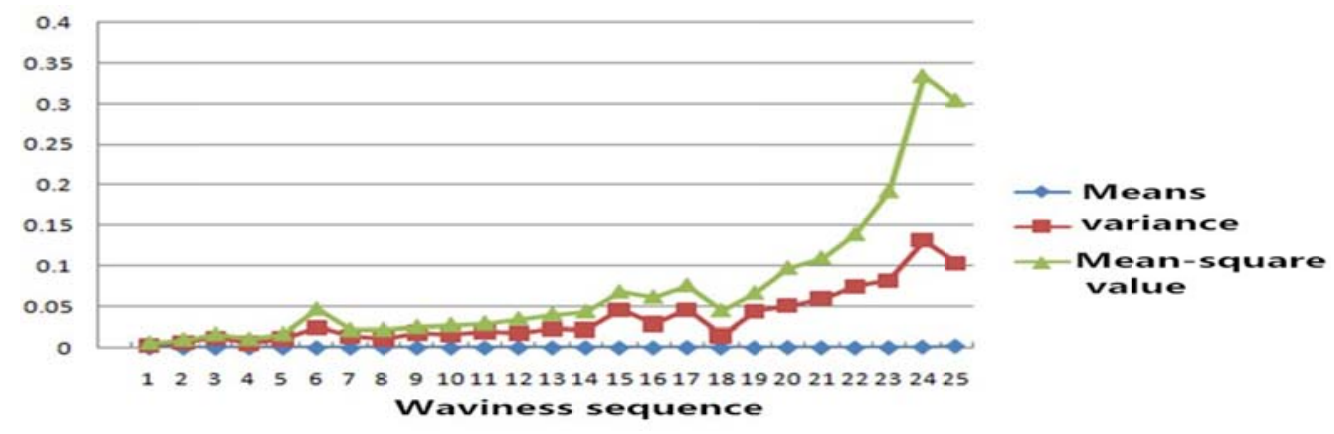

Fig.3 The relationship between statistics characteristics and waviness

To analyze Figure 3, we can see: in KDP crystal SPDT process, when KDP crystal waviness increases, the time-domain characteristics of AE signals mean not show regular changes; With the 
increase of waviness value, variance have an overall increasing trend, but there are jump discontinuity; When you exclude a specific case, the mean-square value and waviness size has a significant correspondence, so it can be used to monitor an important characteristic quantity in the process of KDP crystal surface waviness status .

\subsection{Frequency domain analysis of acoustic emission signals}

Before analyzing the frequency domain, it should be removing a DC component in the time domain at first. [7-9]Then, the time domain signal to Fourier transform use MATLAB, obtain frequency spectrum, and analyze the power spectral of acoustic emission signal, figure 4 shows the grade 1-4 AE signal power spectrum.

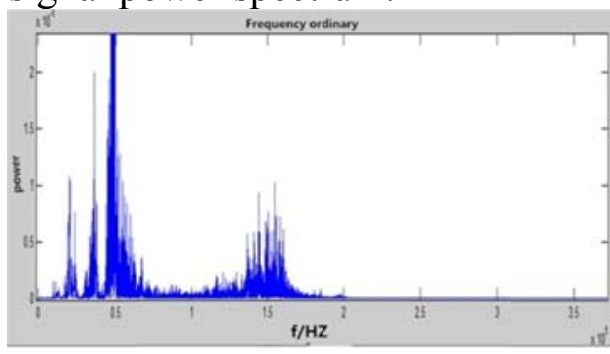

a) Level 1

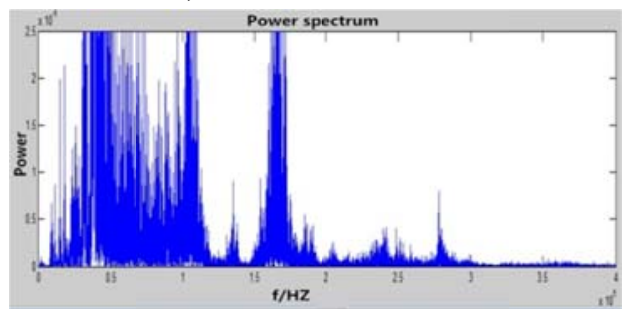

c) Level 3

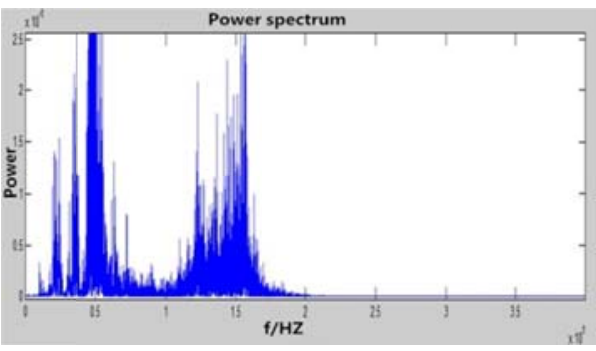

b) Level 2

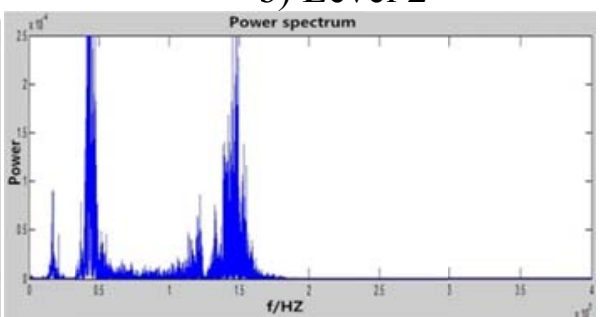

d) Level 4

Fig.4 The power spectrum chart of different waviness grade of AE signal

Figure 4 shows, acoustic emission signals are mainly distributed in $300 \mathrm{KHz}$ or less, $0-300 \mathrm{KHz}$ concentrated in the two signal segments in memory: The large magnitude of the first signals in the middle and stable near 50KHZ; frequency of the second signal segment and focus fluctuation.The signal of the second frequency concentrated segments varies with KDP crystal surface waviness values .And its ripple amplitude increased significantly with increasing values .

By integrating the power spectrum value on each filter band, the average energy obtained as a signal characteristic of this band.Statistics this average of 10 sample length in different frequency bands, as shown in picture 5 .

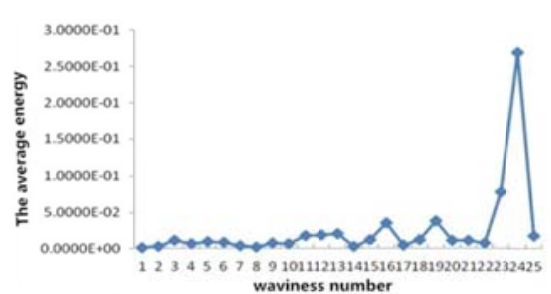

a) $0-50 \mathrm{KHz}$

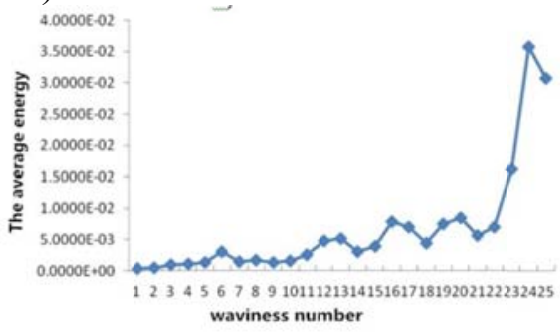

c) $100-150 \mathrm{KHz}$

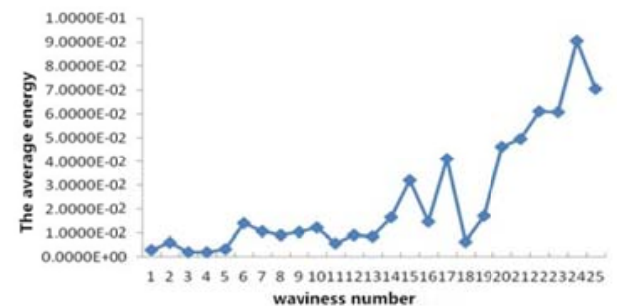

b) $50-100 \mathrm{KHz}$

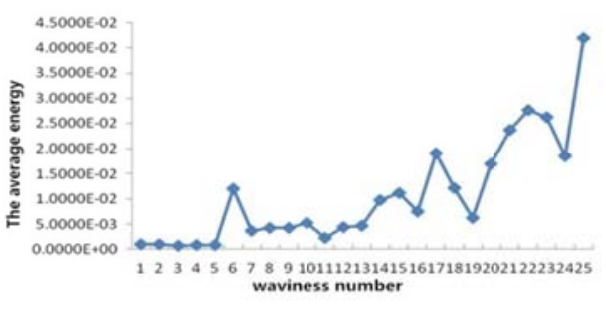

d) $150-200 \mathrm{KHz}$ 


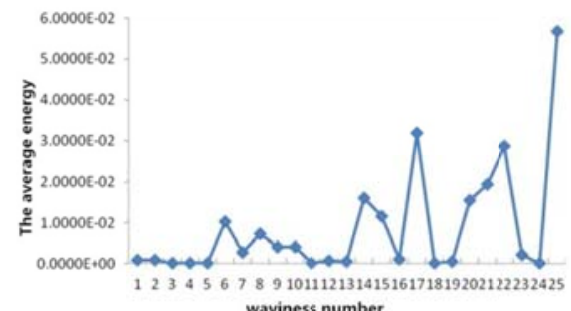

e) $200-250 \mathrm{KHz}$

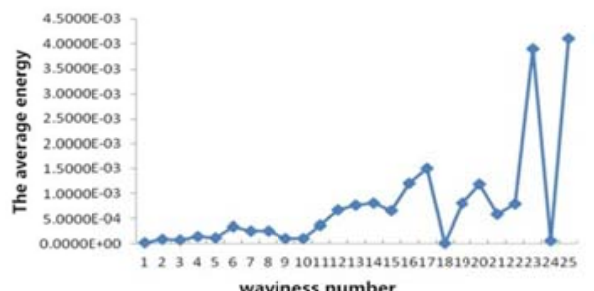

f) $250-300 \mathrm{KH}$

Fig. 5 The relationship between average energy of each band and waviness

The relationship between each band of acoustic emission signal average energy and waviness in picture 5 shows that:with the increase in the value of waviness, the average energy of three bands $50-100 \mathrm{KHz}, 100-150 \mathrm{KHz}$ and $150-200 \mathrm{KHz}$ show the overall increasing trend .The corresponding relationship between the average energy $100-150 \mathrm{KHz}$ and band surface waviness value is the most obvious. The other three band average energy AE signal line chart disorganized, there is no corresponding relationship between surface waviness value.In summary, we can get $150-200 \mathrm{KHz}$ to judge the characteristic surface waviness state band in the cutting process of KDP crystal with single point diamond knife .

\section{Summary}

(1)Adding up the time Domain feature of AE signals at different surface waviness state ,analyzing relationship with waviness, means $\mu_{x}$, variance ${ }^{\sigma_{x}{ }^{2}}$ and Mean-square value ${ }^{{ }^{2}}{ }^{2}$.so Mean-square value $\psi_{x}{ }^{2}$ can be used an important time Domain feature to monitor the process of KDP crystal surface waviness status .

(2)Based on MATLAB software,estimate power spectrum of acoustic emission signal and filter 6 bands of acoustic emission signal,then get the average energy of different bands by statistics.Analyze the change of the average energy value increasing with waviness, get that $150-200 \mathrm{KHz}$ can be used an important band characteristics to monitor KDP crystal surface waviness status .

Foundation Project, National key S\&T Special Projects, Special Crystal Material Single Point Diamond Fly Knife Cutting Ultra-Precision Machine Tools（2011ZX04004-042）

\section{References}

[1]SU Gen-bo, ZENG Jin-bo, HE You-ping, et al. Application of large section KDP crystals in the study of laser fusion [J].Journal of the Chinese Ceramic Society.1997, 25(6):717-719.

[2]YANG Fu-xing.Study on the Ultra-precision Machining Technology for KDP crystals [ $J$ ]. Technology and Test. 2009,9:63-65

[3]JIANG Wei, CHEN Ming-jun. Experimental Investigation of Process Technology for Surface Quality of KDP Crystal Machined by Single Point Diamond Turning [ J ]. Aviation Precision manufacturing Technology, 2009,45(5):4-7

[4]MA En-cai. Research on Mechanical Properties of KDP crystal in Ultra-precision Machined Surface [D]. Harbin: Harbin Institute of technology, 2006:1-5.

[5]WANG Jin-he, WANG Hong-xiang, BI Ting, et al. Forecasting and experimental research of surface roughness in single point diamond turning for KDP crystal [J]. Optical Technique, 2006, 32(2):267-269.

[6] EncaiMa. Research on Mechanical Properties of KDP Crystal in Ultra-precision Machined Surface.[D]. Harbin : Harbin Institute of Technology.2006: 1-5

[7] FengShan , Bao-fengShan. Study on the application for the quadratic general rotary unitized 
design in grain lation[J]. Machinery Design \& Manufacture,2000, (3):64-65

[8] Lu-quanRen. Regression design and optimization[M].Beijing: Science Press, 2009: 1-7.

[9] YingmingXie, XinzhengLi , BinZhang. Progress in the research of KDP crystals[J]. Hebei Journal of Industrial Science and Technology. 2006, (6) : 377-380. 